\title{
Quantification of intranodal vascularity by computer pixel- counting method enhances the accuracy of ultrasound in distinguishing metastatic and tuberculous cervical lymph nodes
}

\author{
Sammy C. H. Cheng ${ }^{1}$, Anil T. Ahuja ${ }^{2}$, Michael Ying ${ }^{1}$ \\ ${ }^{1}$ Department of Health Technology and Informatics, The Hong Kong Polytechnic University, Hong Kong SAR, China; ${ }^{2}$ Department of Imaging and \\ Interventional Radiology, The Chinese University of Hong Kong, Prince of Wales Hospital, Hong Kong SAR, China
}

Correspondence to: Dr. Michael Ying. Department of Health Technology and Informatics, The Hong Kong Polytechnic University, Hong Kong SAR, China. Email: htmying@polyu.edu.hk.

Background: Ultrasound is a common imaging method for assessment of cervical lymph nodes. However, metastatic and tuberculous lymph nodes have similar sonographic features in routine ultrasound examination. Computer-aided assessment could be a potential adjunct to enhance the accuracy of differential diagnosis.

Methods: Gray-scale and power Doppler sonograms of 100 patients with palpable cervical lymph nodes were reviewed and analyzed (60 metastatic nodes, 40 tuberculous nodes). Final diagnosis of lymph nodes was based on fine needle aspiration and cytology. Sonograms were reviewed and assessed for nodal shape, echogenic hilus, intranodal necrosis and vascular distribution (conventional assessment). Intranodal vascularity was quantified using a customized computer algorithm to determine vascularity index (VI). The diagnostic accuracy of using conventional assessment and its combination with intranodal VI method was evaluated and compared.

Results: Metastatic and tuberculous nodes tended to be round (75.0\% vs. 50.0\%), without echogenic hilus ( $86.7 \%$ vs. $72.5 \%)$ and have peripheral vascularity $(73.3 \%$ vs. $85.0 \%)$. Intranodal necrosis is more common in tuberculous nodes $(27.5 \%)$ than metastatic nodes (8.3\%). Using conventional assessment in differentiating metastatic and tuberculous nodes, the diagnostic accuracy was $56 \%$ with a sensitivity, specificity, positive predictive value (PPV) and negative predictive value (NPV) of 56.7\%, 55\%, 65.4\% and 45.8\% respectively. The VI of metastatic nodes $(23.4 \% \pm 2.1 \%)$ was significantly higher than that of tuberculous nodes $(12.0 \% \pm 1.6 \%)(\mathrm{P}<0.05)$. The optimum cut-off of VI for the differential diagnosis was $20 \%$. By combining conventional assessment and intranodal VI quantification, the diagnostic accuracy was increased to $69 \%$ with a sensitivity, specificity, PPV and NPV of 80\%, 52.5\%, 71.6\%, 63.6\% respectively. The increase in sensitivity was statistically significant $(\mathrm{P}=0.006)$.

Conclusions: Computer-aided quantification of intranodal vascularity provides added value in routine ultrasound assessment of cervical lymph nodes. It enhances the accuracy of ultrasound in distinguishing metastatic and tuberculous cervical lymph nodes.

Keywords: Computer-assisted image processing; ultrasonography; lymph nodes; lymphatic metastasis; tuberculosis

Submitted Jun 24, 2019. Accepted for publication Sep 28, 2019.

doi: $10.21037 /$ qims.2019.10.02

View this article at: http://dx.doi.org/10.21037/qims.2019.10.02 


\section{Introduction}

Patients with tuberculous lymphadenitis and head and neck cancers often present with palpable neck lymph nodes. Accurate identification of metastatic neck lymph nodes is vitally important in patients with head and neck carcinoma because it helps estimate the prognosis and aids treatment planning (1-3). Regardless of the primary carcinoma, the presence of unilateral metastatic node reduces the 5-year survival rate to $50 \%$ compare to that of a patient without metastatic node, whereas the survival rate further reduces to $25 \%$ in those with bilateral metastatic lymph nodes $(4,5)$. Tuberculosis is still common in some developing and Southeast Asia countries. In Western countries, tuberculosis can be found in patients who emigrated from countries where the disease is endemic, and in acquired immune deficiency syndrome (AIDS) patients $(6,7)$. Correct identification and differentiation of tuberculous and metastatic lymph nodes are crucial because accurate diagnosis helps the patients to have timely and appropriate treatment.

High-resolution ultrasound has been widely used in cervical lymph node assessment. It is more sensitive than neck palpation alone in detecting cervical lymphadenopathy $(8,9)$. Gray-scale ultrasound could effectively evaluate the distribution and the morphology of cervical lymph nodes such as the size, shape, borders, matting and the internal architecture (10), whilst colour and power Doppler ultrasound are routinely employed to assess intranodal vascularity and vascular resistance $(10,11)$.

Although the role of gray-scale ultrasound on cervical lymph node assessment is well established and the sonographic appearance of different lymphadenopathy has been documented, the sonographic features of tuberculous nodes are variable and simulating metastatic nodes leading to misdiagnosis. A previous study used a computer algorithm to quantify the intranodal vascularity in Doppler ultrasound images, and found that using the quantified intranodal vascularity index (VI) is useful in differentiating between tuberculous and metastatic lymph nodes (12). However, the added value of intranodal VI on routine ultrasound assessment is unknown. Therefore, this study aimed to investigate the added value of this new computeraided method on the routine ultrasound assessment in distinguishing between metastatic and tuberculous cervical lymph nodes.

\section{Methods}

This study was approved by the Human Subject Ethics Subcommittee of the authors' institution. The study procedures conformed to the provisions in accordance with the Helsinki Declaration. Gray-scale and power Doppler sonograms of 100 patients with fine needle aspiration and cytology (FNAC) proven metastatic and tuberculous cervical lymph nodes were retrospectively reviewed. Among the 100 patients, 60 patients had known head and neck cancers or primary tumours in other body regions with cervical lymph node metastasis (15 nasopharyngal carcinomas, 15 oral cavity carcinomas, 10 lung carcinomas, 8 pharyngeal and laryngeal carcinomas, 8 papillary thyroid carcinomas, 2 colon carcinomas and 2 breast carcinomas), whilst 40 patients had known tuberculous lymph nodes and without known malignancy. These 100 patients were consecutively selected from our patient database. Patients without any confirmed pathology or cytology results and those received radiotherapy were excluded from the study.

The same operator, who had more than 30 years of experience in neck ultrasound, performed the neck ultrasound examination of the patients using the same ultrasound scanning protocol, whilst another operator carried out the image analysis. The operator was blinded to the pathology and cytology results during the time of image analysis.

All ultrasound examinations were conducted using a Philips IU22 ultrasound unit in conjunction with a 5 to $12 \mathrm{MHz}$ linear transducer (Philips, Bothell, WA, USA). Both gray-scale and power Doppler ultrasound were used to evaluate cervical lymph nodes, and the lymph node of each patient with the highest vascularity was included in the study. Standardized settings for power Doppler ultrasound was employed for the detection of intranodal blood vessels which include high sensitivity, low wall filter, pulsed repetition frequency of $700 \mathrm{~Hz}$ and medium persistence. The colour gain was firstly increased in the beginning to show colour noise and then gradually decreased until the noise dissipated (13).

On the gray-scale ultrasound, the cervical lymph nodes were evaluated for their shape and internal architecture. The lymph node shape was determined by the short-tolong axis ratio $(\mathrm{S} / \mathrm{L})$. The $\mathrm{S} / \mathrm{L}$ ratio greater than or equal to 0.5 implied a round node, whilst the ratio smaller than 0.5 implied a long or oval node. The internal architecture 
of lymph node was evaluated for the presence or absence of echogenic hilus and cystic necrosis. The echogenic hilus was identified as a hyperechoic linear structure that was continuous with the adjacent soft tissue. Cystic necrosis was demonstrated as focal hypoechoic area(s) within the lymph node.

Multiple Doppler ultrasound images were taken at different parts of the lymph node and the image with the highest intranodal vascularity was selected for evaluation. Settings of Doppler ultrasound were standardized and set for high sensitivity, with a low wall filter to allow detection of blood vessels with low blood flow. The pulse repetition frequency (PRF) was $500 \mathrm{~Hz}$. The colour gain setting was standardized by initially increasing the gain until the colour noise was apparent, and then gradually decreasing unit the noise was just disappeared. Different from the vascular pattern classification in previous studies which is more detailed and has eight categories $(14,15)$, in the present study the vascular patterns of lymph nodes were divided into four categories depending on the distribution of vascularity (13):

(I) Hilar: flow signals diverging radially from the hilus and not along the periphery of the lymph node;

(II) Peripheral: flow signals along the periphery of the lymph node but not arising from the hilum, with branches perforating the periphery of the node;

(III) Mixed: presence of both hilar and peripheral flow signals;

(IV) Apparently avascular: without any vascular signals within the lymph node.

In the part I of the study (conventional assessment), lymph nodes were considered to be metastasis if they demonstrated at least three of the following malignant ultrasound features, otherwise the lymph nodes were considered to be tuberculous nodes:

(I) Short axis to long axis (S/L) ratio $\geq 0.5$;

(II) Absence of echogenic hilus;

(III) Presence of intranodal necrosis;

(IV) Presence of peripheral or mixed vascular patterns.

In the part II of the study, VI (degree of vascularity) of each lymph node was obtained. For each ultrasound image, the degree of intranodal vascularity was assessed by a customized algorithm using the software MATLAB (Version 9.1.0.441655 R2016b, The MathWorks, Natick, MA, USA). In the assessment of lymph node vascularity, the boundary of the lymph node (i.e., the region of interest, ROI) was outlined manually on the ultrasound image in Microsoft Paint (Version 1709, Microsoft, Redmond, WA,
USA). The ROI was extracted from the ultrasound image and the total number of pixels of the ROI was measured by the customized algorithm. Eventually, the colour pixels (i.e., the pixels other than gray-scale) were extracted from the ROI and the number of colour pixels was recorded (Figures 1,2). The VI (in percentage) of each lymph node was then calculated by the following equation (16):

$\mathrm{VI}=($ No. of colour pixels within the ROI $) /($ Total number of pixels within the ROI) $\times 100 \%$

An unpaired $t$-test was used to calculate the significance of difference in VI between metastatic and tuberculous lymph nodes. Receiver operating characteristic (ROC) curve was used to determine the optimum cut-off of VI in differentiating metastatic and tuberculous lymph nodes, and the associated sensitivity and specificity of the optimum cut-off. All statistical analyses were performed using the Statistical Package for the Social Sciences (SPSS) software (Version 20, IBM Corporation, Armonk, NY, USA). A P value $<0.05$ indicated statistical significance.

To evaluate the diagnostic performance in combining conventional assessment method and VI analysis, lymph nodes were considered to be metastasis if they demonstrated at least three of the abovementioned malignant ultrasound features or the VI $\geq$ the optimum cut-off value, otherwise the lymph nodes were considered to be tuberculosis:

The sensitivity, specificity, positive predictive value (PPV), negative predictive value (NPV) and diagnostic accuracy in the differential diagnosis in part I and II studies were evaluated and compared. Chi square test was used to calculate the significance of difference of diagnostic performance between conventional assessment and the combination of conventional assessment and VI analysis.

\section{Results}

In the part I of the study, the lymph node was considered to be metastatic if it showed three out of four predetermined sonographic appearances on its gray-scale and power Doppler sonograms, otherwise was considered to be a tuberculous node. The sonographic features of metastatic and tuberculous lymph nodes are shown in Table 1. Using the conventional assessment method based on the grayscale ultrasound features and intranodal vascular pattern, the sensitivity, specificity, PPV, NPV and diagnostic accuracy in differentiating metastatic and tuberculous nodes were $56.7 \%$, $55 \%, 65.4 \%, 45.8 \%$ and $56 \%$, respectively (Figures 3,4).

In the part II of the study, the intranodal VI of each lymph node was also evaluated. The mean VI 

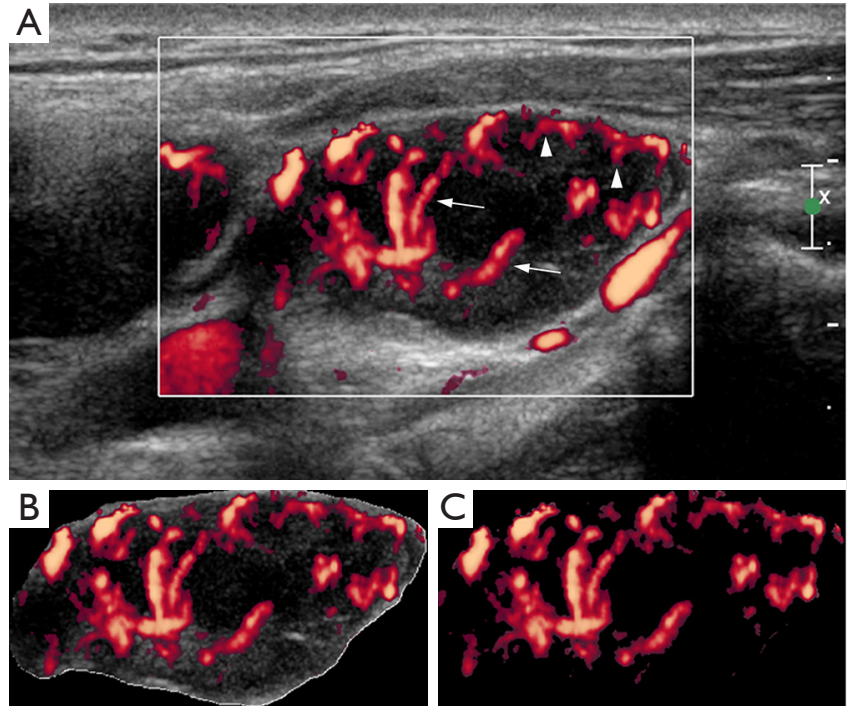

Figure 1 The sequence of image analysis of the power Doppler sonogram of a metastatic lymph node. (A) The original ultrasound image with the lymph node showed both hilar (arrows) and peripheral (arrowheads) vascularity. (B) The image with the region of interest, ROI (i.e., the lymph node) extracted from the ultrasound image, and the total number of pixels within the ROI was counted by the computer algorithm. (C) The image with the colour pixels (coded by the power Doppler ultrasound) extracted by eliminating the grey scale pixels, and the number of colour pixels was counted by the computer algorithm. The calculated vascularity index of this lymph node was $36.6 \%$.

of the metastatic nodes was $23.4 \% \pm 2.1 \%$, which was significantly higher than that of the tuberculous nodes $(12.0 \% \pm 1.6 \%)(\mathrm{P}=0.0001$, Figures 1,2$)$. A ROC curve was plotted to determine the diagnostic performance of VI in distinguishing metastatic nodes from tuberculous nodes. The ROC curve showed that the optimum cut-off of VI in differentiating between metastatic and tuberculous nodes was $20 \%$ with a sensitivity of $58.3 \%$, specificity of $82.5 \%$ and area under the curve (AUC) of 0.71 (Figure 5). In this part of the study, a lymph node was considered to be metastatic if it showed at least three of the malignant ultrasound features, or the VI was greater than or equal to the optimum cut-off. Using these assessment criteria, the sensitivity, specificity, PPV, NPV and diagnostic accuracy in differentiating metastatic and tuberculous nodes were $80 \%$, $52.5 \%, 71.6 \%, 63.6 \%$ and $69 \%$, respectively.

Considered the results of parts I and II of the study, there was increase in the sensitivity (56.7\% to $80 \%)$, PPV (65.4\% to $71.6 \%)$, NPV (45.8\% to $63.6 \%$ ) and overall diagnostic
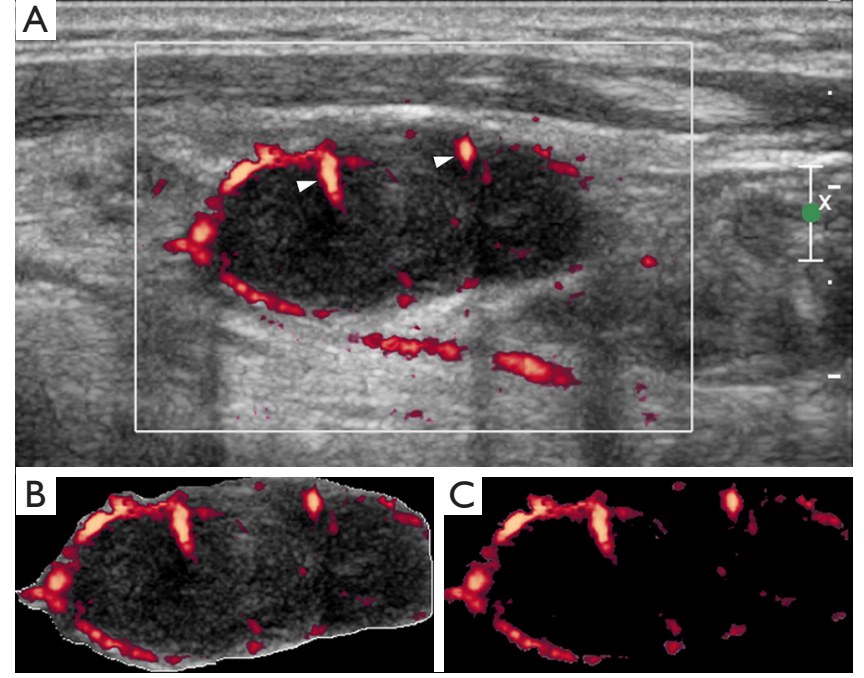

Figure 2 The sequence of image analysis of the power Doppler sonogram of a tuberculous lymph node. (A) The original ultrasound image, and the lymph node showed peripheral vascularity (arrowheads). The steps of the image analysis are the same as described in Figure 1. The calculated vascularity index of this lymph node was $15.1 \%$.

accuracy $(56 \%$ to $69 \%)$ whereas only slight decrease in the specificity (55\% to $52.5 \%$ ), after combining intranodal VI quantification with the conventional assessment. Statistically, the increase in sensitivity was significant $(\mathrm{P}=0.006)$, whereas the differences in specificity, PPV and NPV were not significant $(\mathrm{P}=0.824,0.471$ and 0.117 respectively). The increase in overall diagnostic accuracy was marginally insignificant $(\mathrm{P}=0.058)$.

\section{Discussion}

Evaluation of lymph node vascularity has been reported. A previous study assessed intranodal vascularity by subjective visual judgement and classified lymph nodes according to the abundance of intranodal vascularity (13). Another study evaluated the diagnostic performance of solely VI analysis of lymph node but without studied the diagnostic performance of combining VI analysis with conventional ultrasound assessment of lymph node (16). In the present study, we have investigated the added value of VI analysis on conventional ultrasound assessment to distinguish between metastatic and tuberculous cervical lymph nodes. In the first part of the study, results showed that the sonographic appearances of metastatic and tuberculous 
Table 1 Comparison of gray-scale and power Doppler sonographic features of metastatic and tuberculous lymph nodes

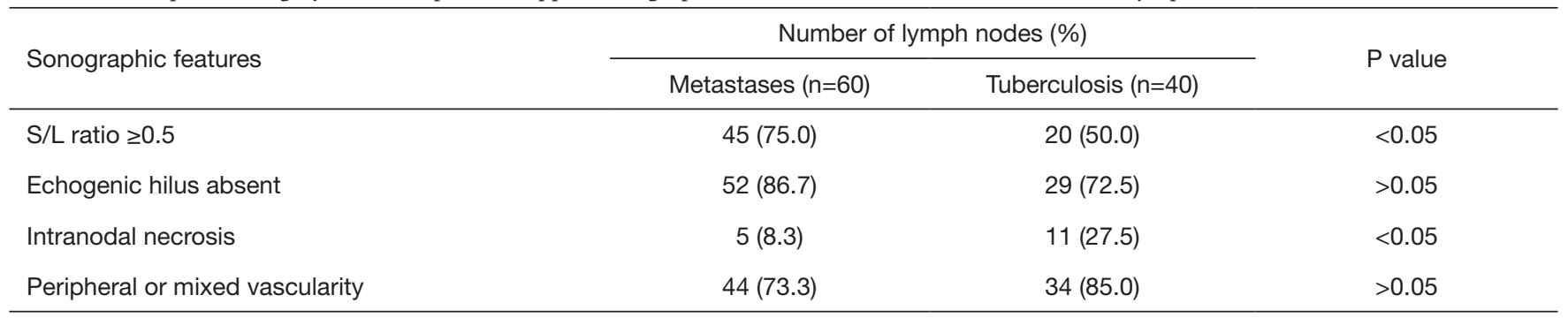

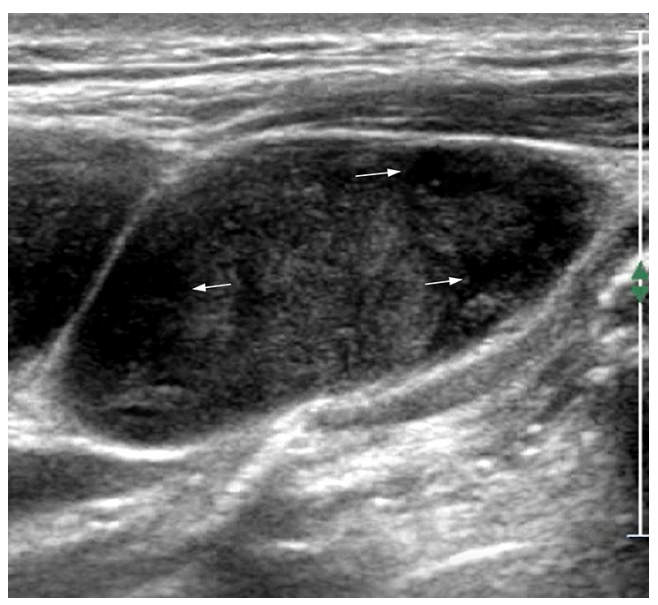

Figure 3 Longitudinal gray-scale sonogram of the same metastatic lymph node as described in Figure 1. The lymph node was hypoechoic, oval in shape, without echogenic hilus and had intranodal necrosis (arrows).

nodes were quite similar. Though intranodal necrosis was more common in the tuberculous nodes $(27.5 \%)$ when compared to metastatic nodes $(8.3 \%)$, both of them tended to be round in shape (metastasis: $75.0 \%$; tuberculosis: 50.0\%), without echogenic hilus (metastasis: $86.7 \%$; tuberculosis $72.5 \%$ ) and had peripheral or mixed vascularity (metastasis: $73.3 \%$; tuberculosis: $85.0 \%$ ). Hence, the diagnostic accuracy of the conventional assessment method (56\%) was not good enough for an accurate diagnosis in differentiating metastatic and tuberculous nodes. This was due to the high false-negative rate of which some metastatic nodes were at the early stage of the disease and did not demonstrate sufficient malignant ultrasound features. In addition, the low diagnostic accuracy was also account for the high false-positive rate of which tuberculous nodes presented with malignant ultrasound features mimicking metastatic nodes. Therefore, using the conventional method of assessing the gray-scale ultrasound features and vascular

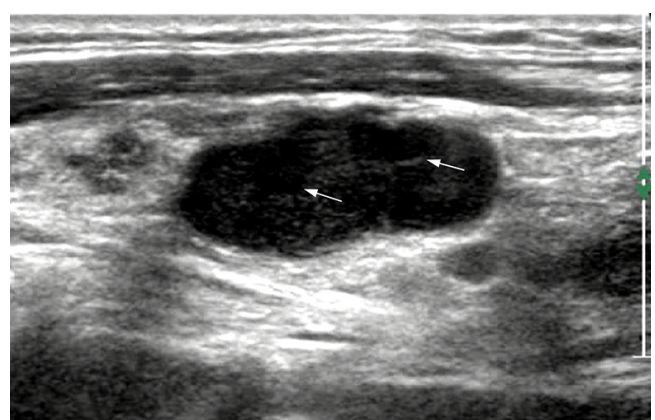

Figure 4 Longitudinal gray-scale sonogram of the same tuberculous lymph node as described in Figure 2. The lymph node was hypoechoic, oval in shape, without echogenic hilus and had intranodal necrosis (arrows). The gray-scale ultrasound features of the tuberculous node were similar to the metastatic lymph node as described in Figure 3.

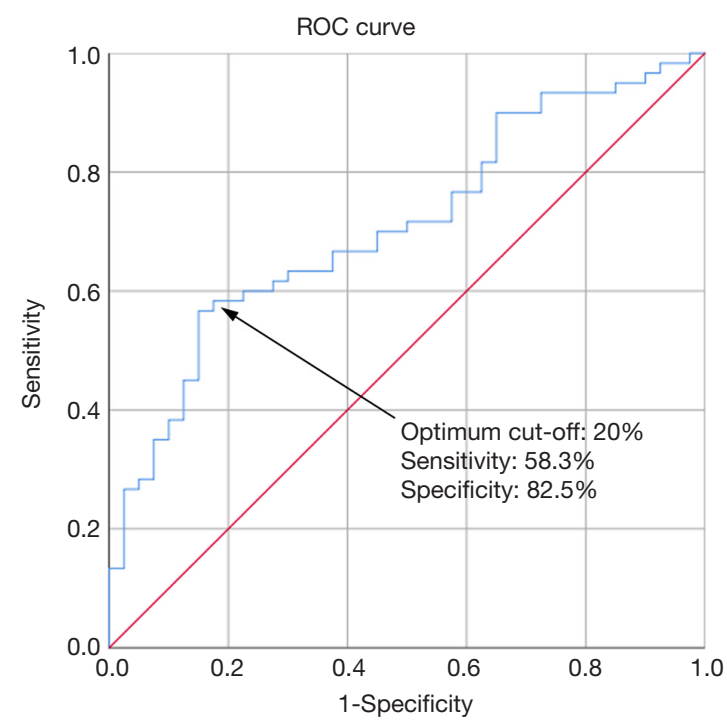

Figure 5 ROC curve of the intranodal vascularity index in differentiating metastatic lymph nodes from tuberculous lymph nodes. 
pattern of lymph nodes, physicians may find difficulty in differential diagnosis for the tuberculosis patients with cancers on routine ultrasound examination, particularly in those regions of the world where the prevalence of tuberculosis is high.

In the second part of the study, quantification of intranodal VI was introduced in order to aid differential diagnosis. VI is a ratio of the number of colour pixels to the total number of pixels within the region of interest (i.e., the lymph node in this study) on a power Doppler sonogram, indicating the degree of vascularity. The results showed that the mean VI of the metastatic nodes (mean $\mathrm{VI}=23.4 \%$ ) was significantly higher than that of the tuberculous nodes (mean VI $=12 \%)(\mathrm{P}<0.05)$. The higher intranodal vascularity of metastatic nodes is likely due to tumour angiogenesis, resulting in increased tumour blood vessels within lymph nodes. On the other hand, higher occurrence of intranodal necrosis in the tuberculous nodes could cause intranodal blood vessels to be displaced by the necrotic tissue, resulting in reduced vascularity. This finding is consistent to our previous study (12) and other previous studies showing apparently avascular pattern and vascularity displacement in tuberculous nodes on Doppler sonograms $(17,18)$. One must note that in the present study the classification of the distribution of intranodal vascularity was qualitative assessment based on human judgement, whereas the quantification of intranodal VI was the quantity of the color pixels in Doppler ultrasound image computed by the customized algorithm.

In the differential diagnosis of metastatic, tuberculous, lymphomatous and reactive lymph nodes, a previous study demonstrated that intranodal VI had the highest accuracy in distinguishing metastatic and tuberculous nodes, suggesting that VI is a useful parameter in distinguishing these two pathological conditions (12). With the use of ROC curve, the present study found that a VI of $20 \%$ was the optimum cut-off to differentiate metastatic nodes from tuberculous nodes, and it yielded a sensitivity and specificity of $58.3 \%$ and $82.5 \%$ respectively in the differential diagnosis. The finding was consistent with a previous study in which the optimum cut-off of intranodal VI in differentiating metastatic and tuberculous lymph nodes was $22 \%$ (12).

Although computer-aided assessment of intranodal VI can objectively evaluate and quantify the intranodal vascularity and is useful in differentiating metastatic and tuberculous nodes, assessment of the internal architecture and vascular distribution of lymph node are also important in routine clinical practice $(8,18)$. Therefore, we investigated the diagnostic value of combining intranodal VI quantification with conventional assessment of gray-scale ultrasound features and vascular pattern of lymph nodes. Results showed that there was a significant increase in the sensitivity, while the specificity remained similar, after combining intranodal VI quantification with the conventional assessment. The improvement of the sensitivity was due to the increased truepositive rate and the associated decrease in false-negative rate after using intranodal VI in the assessment. Tumour angiogenesis usually occurs before there is any substantial morphological change of the tumour (19). Some early-staged metastatic lymph nodes might not demonstrate malignant gray-scale ultrasound features such as absent echogenic hilus, round in shape and intranodal necrosis because these features are associated with extensive tumour infiltration within the lymph node. However, tumour angiogenesis could occur in these lymph nodes leading to increased intranodal VI. Therefore, metastatic lymph nodes were not identified in conventional assessment method, which was predominantly based on gray-scale ultrasound features, was detected in the intranodal VI assessment yielding higher true-positive rate. The slight decrease in the specificity was due to one tuberculous node was considered as true-negative case in conventional assessment but had an intranodal VI greater than $20 \%$ leading to slight increase in the falsepositive rate.

The results of the current study showed that tuberculous nodes tended to have lower intranodal VI when compared to metastatic nodes. The lower VI in tuberculous nodes is likely due to the high incidence of intranodal necrosis in these lymph nodes which displaced the intranodal vasculature. However, one must note that intranodal necrosis is also common in metastatic nodes from squamous cell carcinoma and papillary carcinoma of the thyroid gland $(8,20,21)$. For that reason, other sonographic features such as intranodal vascular distribution, shape, matting, existence of calcification and adjacent soft tissue edema should also be considered to identify the pathologic condition of lymph nodes $(18,22,23)$.

The present study reported a computer-aided method to quantify the intranodal VI, and found that combined with the conventional assessment method can improve the diagnostic accuracy in differentiating metastatic and tuberculous lymph nodes. In the computer-aided analysis of Doppler ultrasound images, it took about 5 minutes to outline one lymph node on the image manually and process the image with the software program. With the availability of deep learning-based algorithm for automated 
segmentation of lymph nodes (24), the time for image processing could be significant reduced. This can allow the computer-aided assessment of intranodal VI to become applicable in routine clinical practice. It is widely known that the assessment of intranodal vascularity in a threedimensional (3-D) way is more accurate than the twodimensional (2-D) method because tumour angiogenesis is developed in a 3-D matrix (25). The present study provided a strong basis on computer-aided quantification of intranodal vascularity on 2-D ultrasound images. Further development of integrating this technology with 3-D ultrasound imaging can enhance the accuracy and reliability in the assessment of intranodal vascularity. Previous studies on analysis of the VI of breast cancer in 3-D ultrasound found that intra-tumour VI is useful in the evaluation of prognosis and treatment response of breast cancer patients $(26,27)$. Recent superb microvascular imaging (SMI) technology allows for detection of microvascularity. Ryoo et al. (28) suggested that assessment of the vascular pattern of lymph nodes using SMI helped the differentiation of metastatic and tuberculous lymph nodes with good interobserver agreement. As microvascularity be detected in SMI, there is a need to have a computer-aided assessment method, similar to the one described in the present study, to accurately and objectively identify the subtle vascularity within lymph nodes.

Our findings demonstrated that computer-aided method provided objective quantification of intranodal vascularity, and gave added value in routine ultrasound assessment of cervical lymph nodes. Quantified intranodal VI can enhance the accuracy of ultrasound in distinguishing metastatic and tuberculous cervical lymph nodes.

\section{Acknowledgments}

Funding: This study is supported by a Departmental General Research Fund of the Hong Kong Polytechnic University (P0008696:G-UADH).

\section{Footnote}

Conflicts of Interest: The authors have no conflicts of interest to declare.

Ethical Statement: This study was approved by the Human Subject Ethics Subcommittee of the authors' institution. The study procedures conformed to the provisions in accordance with the Helsinki Declaration.

\section{References}

1. Baatenburg de Jong RJ, Rongen RJ, Lameris JS, Harthoorn M, Verwoerd CD, Knegt P. Metastatic neck disease. Palpation vs ultrasound examination. Arch Otolaryngol Head Neck Surg 1989;115:689-90.

2. Ishii J, Amagasa T, Tachibana T, Shinozuka K, Shioda S. US and CT evaluation of cervical lymph node metastasis from oral cancer. J Craniomaxillofac Surg 1991;19:123-7.

3. Vassallo P, Wernecke K, Roos N, Peters PE. Differentiation of benign from malignant superficial lymphadenopathy: the role of high-resolution US. Radiology 1992;183:215-20.

4. Som PM. Detection of metastasis in cervical lymph nodes: CT and MR criteria and differential diagnosis. AJR Am J Roentgenol 1992;158:961-9.

5. Lydiatt WM, Patel SG, O'Sullivan B, Brandwein MS, Ridge JA, Migliacci JC, Loomis AM, Shah JP. Head and Neck cancers-major changes in the American Joint Committee on cancer eighth edition cancer staging manual. CA Cancer J Clin 2017;67:122-37.

6. Agarwal M, Nabavizadeh SA, Mohan S. Chapter 6 NonSquamous Cell Causes of Cervical Lymphadenopathy. Semin Ultrasound CT MR 2017;38:516-30.

7. Global tuberculosis report 2018. Geneva: World Health Organization; 2018.

8. Giacomini CP, Jeffrey RB, Shin LK. Ultrasonographic evaluation of malignant and normal cervical lymph nodes. Semin Ultrasound CT MR 2013;34:236-47.

9. Haberal I, Celik H, Gocmen H, Akmansu H, Yoruk $\mathrm{M}$, Ozeri C. Which is important in the evaluation of metastatic lymph nodes in head and neck cancer: palpation, ultrasonography, or computed tomography? Otolaryngol Head Neck Surg 2004;130:197-201.

10. Ying M, Bhatia KS, Lee YP, Yuen HY, Ahuja AT. Review of ultrasonography of malignant neck nodes: greyscale, Doppler, contrast enhancement and elastography. Cancer Imaging 2014;13:658-669.

11. Ariji Y, Kimura Y, Hayashi N, Onitsuka T, Yonetsu K, Hayashi K, Ariji E, Kobayashi T, Nakamura T. Power Doppler sonography of cervical lymph nodes in patients with head and neck cancer. Am J Neuroradiol 1998;19:303-7.

12. Ying $M$, Cheng SC, Ahuja AT. Diagnostic Accuracy of Computer-Aided Assessment of Intranodal Vascularity in Distinguishing Different Causes of Cervical Lymphadenopathy. Ultrasound Med Biol 2016;42:2010-6. 13. Ying M, Ahuja A, Brook F, Metreweli C. Power Doppler 
sonography of normal cervical lymph nodes. J Ultrasound Med 2000;19:511-7.

14. Tschammler A, Heuser B, Ott G, Schmitt S, Hahn D. Pathological angioarchitecture in lymph nodes: underlying histopathologic findings. Ultrasound Med Biol 2000;26:1089-97.

15. Tschammler A, Wirkner H, Ott G, Hahn D. Vascular patterns in reactive and malignant lymphadenopathy. Eur Radiol 1996;6:473-80.

16. Lam J, Ying M, Cheung SY, Yeung KH, Yu PH, Cheng HC, Ahuja AT. A Comparison of the Diagnostic Accuracy and Reliability of Subjective Grading and Computer-Aided Assessment of Intranodal Vascularity in Differentiating Metastatic and Reactive Cervical Lymphadenopathy. Ultraschall Med 2016;37:63-7.

17. Ahuja A, Ying M, Yuen YH, Metreweli C. Power Doppler Sonography to Differentiate Tuberculous Cervical Lymphadenopathy from Nasopharyngeal Carcinoma. Am J Neuroradiol 2001;22:735-40.

18. Park JH, Kim DW. Sonographic diagnosis of tuberculous lymphadenitis in the neck. J Ultrasound Med 2014;33:1619-26.

19. Bielenberg DR, Zetter BR. The Contribution of Angiogenesis to the Process of Metastasis. Cancer J 2015;21:267-73.

20. Kessler A, Rappaport Y, Blank A, Marmor S, Weiss J, Graif M. Cystic appearance of cervical lymph nodes is characteristic of metastatic papillary thyroid carcinoma. J Clin Ultrasound 2003;31:21-5.

21. Ustün M, Risberg B, Davidson B, Berner A. Cystic change in metastatic lymph nodes: a common diagnostic pitfall in fine-needle aspiration cytology. Diagn Cytopathol

Cite this article as: Cheng SCH, Ahuja AT, Ying M. Quantification of intranodal vascularity by computer pixelcounting method enhances the accuracy of ultrasound in distinguishing metastatic and tuberculous cervical lymph nodes. Quant Imaging Med Surg 2019;9(11):1773-1780. doi: 10.21037/qims.2019.10.02
2002;27:387-92.

22. Ahuja A, Ying M. Sonography of neck lymph nodes. Part II: abnormal lymph nodes. Clin Radiol 2003;58:359-66.

23. Rosário PW, de Faria S, Bicalho L, Alves MF, Borges MA, Purisch S, Padrao EL, Rezende LL, Barroso AL. Ultrasonographic differentiation between metastatic and benign lymph nodes in patients with papillary thyroid carcinoma. J Ultrasound Med 2005;24:1385-9.

24. Zhang Y, Ying MTC, Yang L, Ahuja AT, Chen DZ. Coarse-to-fine stacked fully convolutional nets for lymph node segmentation in ultrasound images. IEEE International Conference on Bioinformatics and Biomedicine (BIBM); 15-18 Dec. 2016; Shenzhen, China

25. Dong FJ, Xu JF, Du D, Jiao Y, Zhang L, Li M, Liu HY, Xiong Y, Luo H. 3D analysis is superior to 2D analysis for contrast-enhanced ultrasound in revealing vascularity in focal liver lesions - A retrospective analysis of 83 cases. Ultrasonics 2016;70:221-6.

26. Shia WC, Chen DR, Huang YL, Wu HK, Kuo SJ. Effectiveness of evaluating tumor vascularization using 3D power Doppler ultrasound with high-definition flow technology in the prediction of the response to neoadjuvant chemotherapy for T2 breast cancer: a preliminary report. Phys Med Biol 2015;60:7763-78.

27. Jiang J, Chen YQ, Xu YZ, Chen ML, Zhu YK, Guan WB, Wang XJ. Correlation between three-dimensional ultrasound features and pathological prognostic factors in breast cancer. Eur Radiol 2014;24:1186-96.

28. Ryoo I, Suh S, You SH, Seol HY. Usefulness of Microvascular Ultrasonography in Differentiating Metastatic Lymphadenopathy from Tuberculous Lymphadenitis. Ultrasound Med Biol 2016;42:2189-95. 